\title{
Classification of Software Risks with Discriminant Analysis Techniques in Software planning Development Process
}

\author{
Abdelrafe Elzamly ${ }^{1}$, Burairah Hussin ${ }^{2}$, Samy S. Abu Naser ${ }^{3}$, Mohamed Doheir ${ }^{4}$ \\ ${ }^{I}$ Department of Computer Science, Faculty of Applied Sciences, Al-Aqsa \\ University, Gaza, Palestine \\ ${ }^{2,4}$ Information and Communication Technology, Universiti Teknikal Malaysia \\ Melaka (UTeM) \\ ${ }^{3}$ Faculty of Engineering \& Information Technology, Al-Azhar University, Gaza, \\ Palestine
}

\begin{abstract}
The aim of this study is to propose the discriminant analysis (DA) techniques to classify and manage risks in software planning development process. These techniques are used to test the controls to each of risks to determine and classify if they are effective in mitigating the occurrence of each risk planning factor. We classified risks to three levels by predicted group membership. Also top ten risks planning software development were mitigated by using controls based on discriminant analysis techniques.
\end{abstract}

Keywords: Risk Management, software Planning Risks, controls, Discriminant Analysis Techniques, predictive risk model

\section{Introduction}

Despite much research and progress in the area of software project management, software development projects still fail to deliver acceptable systems on time and within budget [1]. Much of the failure could be avoided by managers pro-actively maintenance and dealing with risk factors rather than waiting for problems to occur and then trying to react. Due to the involvement of risk management in monitoring the success of a software project, analyzing potential risks, and making decisions about what to do about potential risks, the risk management is considered the planned control of risk. Integrating formal risk management with project management is a new phenomenon in software engineering and product management community. In addition, risk management methodology that has five phases: Risk identification, risk analysis and evaluation, risk treatment, risk controlling, risk communication and documentation relied on three categories or techniques as risk qualitative analysis, risk quantitative analysis and risk mining analysis throughout the life of a software project to meet the goals [2]. Today, we must think of risk is a part of software project process and is important for a software project survival. Risk management is a practice of controlling risk and practice consists of processes, methods, and tools for managing risks in a software project before they become problems [3]. The objective of this study is: To classify the software planning risks of software development in the software development organizations, to model the activities performed for mitigating the risks planning software development which identified.

\section{Literature Review}

Khanfar et al., [4], the new technique used the chi-square $(\chi 2)$ test to control the risks in a software project. However, we also used new techniques which are the 
regression test and effect size test proposed to manage the risks in a software project and reducing risk with software process improvement [5]. Also we improved quality of software projects of the participating companies while estimating the qualityaffecting risks in IT software projects. The results show that there were 40 common risks in software projects of IT companies in Palestine. The amount of technical and non-technical difficulties was very large [6]. Furthermore, we used the new stepwise regression technique to manage the risks in a software project. These tests were performed using regression analysis to compare the controls to each of the risk factors to determine if they are effective in mitigating the occurrence of each risk factor implementation phase [7]. In addition, we proposed the new mining technique that uses the fuzzy multiple regression analysis techniques to manage the risks in a software project. However, these mining tests were performed using fuzzy multiple regression analysis techniques to compare the risk management techniques to each of the software risk factors to determine if they are effective in mitigating the occurrence of each software risk factor[8]. This paper aimed to present new techniques to determine if fuzzy and stepwise regression are effective in mitigating the occurrence software risk factor in the implementation phase [9]. Additionally, we proposed artifact model of the software risk management for mitigating risks. It has the five levels to mitigate risks through software project [10]. Previous studies had shown that risk mitigation in software projects classified into three categoriesnamely, qualitative, quantitative, and mining approaches. Firstly, quantitative risk is based on statistical methods that deal with accurate measurement about risk or leading to quantitative inputs that helped to form a regression model to understand how software project risk factors influence project success. Furthermore, qualitative risk techniques lead to subjective opinions expressed or self-judgment by software manager using techniques namely scenario analysis, Delphi analysis, brainstorming session, and other subjective approach to mitigate risks. There are several software risk management approaches, models, and framework according to a literature review.

\section{Top 10 Software Planning Risks in Software Development Process:}

We displayed the top software planning risk factors in software development project lifecycle that most common used by researchers when studying the risk in software projects. However, the list consists of the 10 most serious risks to a project ranked from one to ten, each risk's status, and the plan for addressing each risk. These factors need to be addressed and thereafter need to be controlled. These software project risks are illustrated [11]: Low key user involvement, Unrealistic schedules and budgets, Unrealistic schedules and budgets, Unclear / misunderstood / Unrealistic / change scope and objectives (goals), Insufficient/inappropriate staffing, Lack of senior management commitment and technical leadership, Poor /inadequate planning and strategic thinking, Lack of effective software project management methodology, Change in organizational management during the software project, Ineffective communication software project system, Absence of and historical data (templates).

\section{Risk control Techniques}

Through reading the existing literature on software risk management approach and methodology, we listed thirty control factors that are considered important in reducing and modeling the software risk factors identified in planning software development; these controls are [12]-[19]: 
$\mathrm{C} 1$ : Using of requirements scrubbing, C2: Stabilizing requirements and specifications as early as possible, C3: Assessing cost and scheduling the impact of each change to requirements and specifications, C4: Develop prototyping and have the requirements reviewed by the client, C5: Developing and adhering a software project plan,C6: Implementing and following a communication plan, C7: Developing contingency plans to cope with staffing problems, C8: Assigning responsibilities to team members and rotate jobs, C9: Have team-building sessions, C10: Reviewing and communicating progress to date and setting objectives for the next phase, C11: Dividing the software project into controllable portions, C12: Reusable source code and interface methods, C13:Reusable test plans and test cases, C14: Reusable database and data mining structures, C15: Reusable user documents early, C16: Implementing/Utilizing automated version control tools, C17: Implement/ utilize benchmarking and tools of technical analysis, C18: Creating and analyzing process by simulation and modeling, C19: Provide scenarios methods and using of the reference checking, C20: Involving management during the entire software project lifecycle, C21:Including formal and periodic risk assessment, C22:Utilizing change control board and exercise quality change control practices, C23: Educating users on the impact of changes during the software project, C24: Ensuring that quality-factor deliverables and task analysis, C25: Avoiding having too many new functions on software projects, C26: Incremental development (deferring changes to later increments), C27: Combining internal evaluations by external reviews, C28: Maintain proper documentation of each individual's work, C29: Provide training in the new technology and organize domain knowledge training, C30: Participating users during the entire software project lifecycle.

\section{Empirical Strategy}

Data collection was achieved through the use of a structured questionnaire for estimating the quality of software through determine risks that were common to the majority of software projects in the analyzed software companies. Top ten software planning risks and thirty control factors were presented to respondents. The method of sample selection referred to as distribution personal regular sampling was used. This procedure is appropriate when members of homogeneous groups (such as software project managers, IT managers) are difficult to locate. The seventy six software project managers have participated in this study. The project managers that participated in this survey are coming from specific mainly software project manager in software development organizations. However to describe "software Development Company in Palestine" that have in-house development software and supplier of software for local or international market, we depended on Palestinian Information Technology Association (PITA) Members' webpage at PITA's website [PITA 2012 www.pita.ps/], Palestinian investment promotion agency [PIPA 2012 http://www.pipa.gov.ps/] to select top IT manager, software project managers. In this paper, we Discriminant Analysis techniques to classify software planning risks in software development process. A Clearly, Discriminant Analysis (DA) is used for predicting group membership based on a linear combination of independent variables. Wilks Lambda $\left({ }^{\wedge}\right)$ is used to test the efficiency of Discriminant Analysis (DA) function. This measure indicates the significant difference between the target groups. The Discriminant function is expressed as follows.

$\mathrm{DF}=\mathrm{w} 1 \mathrm{C} 1+\mathrm{w} 2 \mathrm{C} 2+\ldots+\mathrm{wi} \mathrm{Ci}+\mathrm{a}$

\section{Relationships between Risks and Control Variables}

These tests are used the discriminant analysis techniques to compare the controls to each of the risk planning software development factors to determine and evaluate 
if they are effective in mitigating the occurrence of each risk factor. Wilk's Lambda is used to test if there is relationship between the discriminant function and the independent variables (controls).

\section{R1: Risk Of 'Low Key User Involvement' Compared To Controls.}

Table 1. Wilks' Lambda

\begin{tabular}{|c|c|c|c|c|}
\hline Test of Function(s) & Wilks' Lambda & Chi-square & Df & Sig. \\
\hline 1 through 2 & .599 & 36.867 & 6 & .000 \\
2 & .923 & 5.777 & 2 & .056 \\
\hline
\end{tabular}

Table 2. Classification Results for Risk 1 using Discriminant Function

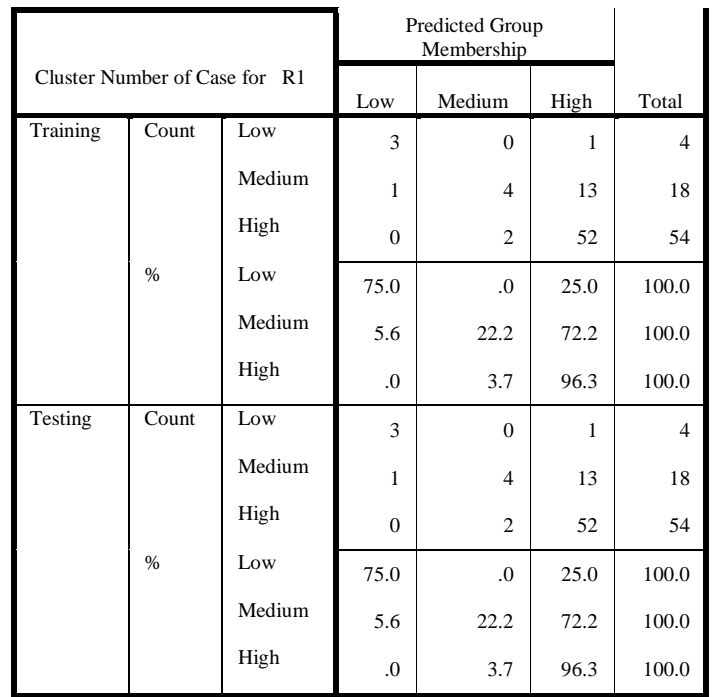

a Cross validation is done only for those cases in the analysis. In cross validation, each case is classified by the functions derived from all cases other than that case. b $77.6 \%$ of original grouped cases correctly classified.

c $77.6 \%$ of cross-validated grouped cases correctly classified.

Table 1 and Table 2 show that discriminant function 1 explains $86.6 \%$ of the variance between the risk groups while discriminant function 2 only accounts for $13.4 \%$ of the variance. Also it indicates the canonical correlation of the discriminant functions to the independent variables. Functions 1 and 2 have positive correlation $(\mathrm{r}=0.592,0.278)$ hence both function 1 is important for the classification of the independent variables to risk groups. Eigen value is significant for the discriminant function 1 . The chi-square values $\left(\chi^{2}=36.867\right)$ which is a statistics for measuring these tests of significance of the Eigen values. However, Wilk's Lambda is used to test if there is relationship between the discriminant function and the independent variables. Associated with each Wilk's Lambda is a chi-square statistics to measure the significance of this relationship. If this chi-square statistic corresponding to Wilk's Lambda is statistically significant it concluded that a relationship exists between the discriminant function and the independent variables. The result shows there is significant relationship between the discriminant function 1 and the independent variables of c1, c21, c28 related groups. The output for significance tests and strength of relationship statistics for the discriminant analysis is shown in Table 1. The coefficients for building the classification models are presented in equation 2. The output for classification of groups is shown in Table 2. The classification results allow us to determine how well we can predict group membership using a classification functions. The results in equation the 
discriminant function equation for predicting the classification of risk 1 with risk management techniques in software devotement project are given as:

$\mathrm{DF} 1=1.635 * \mathrm{C} 1+1.336 * \mathrm{C} 21+0.649 * \mathrm{C} 28-10.085$

R2: Risk of 'Unrealistic Schedules and Budgets' Compared to Controls.

Table 3. Wilks' Lambda

\begin{tabular}{|l|l|l|l|l|}
\hline Test of Function(s) & Wilks' Lambda & Chi-square & Df & Sig. \\
\hline 1 & .784 & 17.737 & 2 & .000 \\
\hline
\end{tabular}

Table 4. Classification Results for risk 2 using Discriminant Function

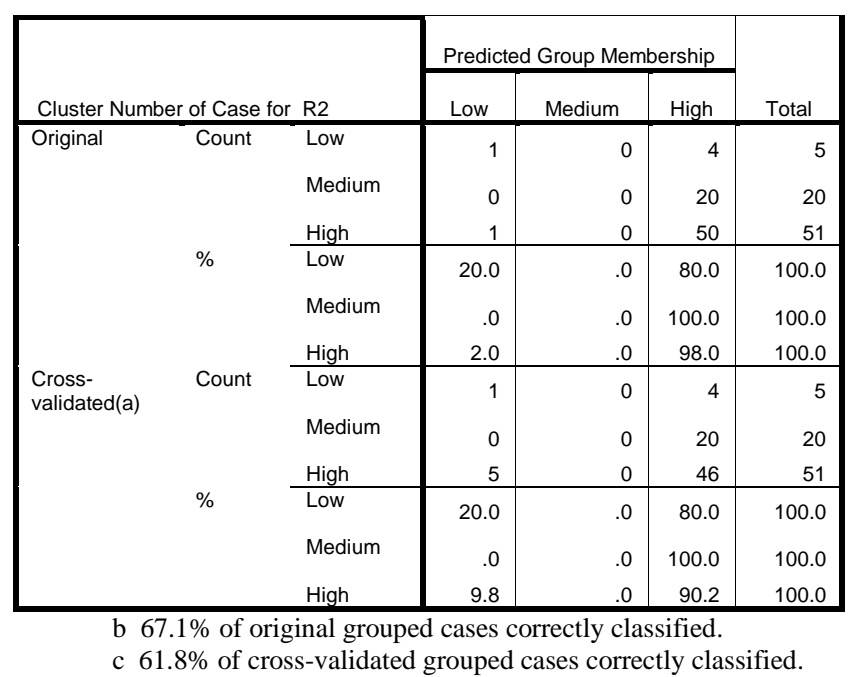

It indicates the canonical correlation of the discriminant functions to the independent variables. Functions 1 has positive correlation $(r=0.464)$, hence function 1 is important for the classification of the independent variables to the risk groups. The chi-square values $(\chi 2=17.737)$ which is a statistics for measuring these tests of significance of the Eigen values. The result shows there is significant relationship between the discriminant function 1 and the independent variables of $c 5$ related groups. The discriminant function equation 1 for predicting the classification of risk 2 with risk management techniques in software devotement project are given as:

$$
\mathrm{DF} 1=2.366 * \mathrm{c} 5-6.599
$$

R3: Risk of 'Misunderstood / Unrealistic Scope and Objectives (Goals)' Compared to Controls.

Table 5. Wilks' Lambda

\begin{tabular}{|l|r|r|r|r|}
\hline Test of Function(s) & Wilks' Lambda & Chi-square & df & Sig. \\
\hline 1 & .892 & 8.336 & 2 & .015 \\
\hline
\end{tabular}


Table 6. Classification Results for risk 3 using Discriminant Function

\begin{tabular}{|c|c|c|c|c|c|c|}
\hline \multirow{2}{*}{\multicolumn{3}{|c|}{ Cluster Number of Case for R3 }} & \multicolumn{3}{|c|}{ Predicted Group Membership } & \multirow[b]{2}{*}{ Total } \\
\hline & & & Low & Medium & High & \\
\hline \multirow[t]{6}{*}{ Original } & \multirow[t]{3}{*}{ Count } & Low & 0 & 1 & 1 & 2 \\
\hline & & Medium & 0 & 8 & 17 & 25 \\
\hline & & High & 0 & 4 & 45 & 49 \\
\hline & \multirow[t]{3}{*}{$\%$} & Low & .0 & 50.0 & 50.0 & 100.0 \\
\hline & & Medium & .0 & 32.0 & 68.0 & 100.0 \\
\hline & & High & .0 & 8.2 & 91.8 & 100.0 \\
\hline \multirow{6}{*}{$\begin{array}{l}\text { Cross- } \\
\text { validated(a) }\end{array}$} & \multirow[t]{3}{*}{ Count } & Low & 0 & 1 & 1 & 2 \\
\hline & & Medium & 0 & 8 & 17 & 25 \\
\hline & & High & 0 & 4 & 45 & 49 \\
\hline & \multirow[t]{3}{*}{$\%$} & Low & .0 & 50.0 & 50.0 & 100.0 \\
\hline & & Medium & .0 & 32.0 & 68.0 & 100.0 \\
\hline & & High & .0 & 8.2 & 91.8 & 100.0 \\
\hline
\end{tabular}

b $69.7 \%$ of original grouped cases correctly classified.

c $69.7 \%$ of cross-validated grouped cases correctly classified.

The canonical correlation of the discriminant functions to the independent variables. Functions 1 has positive correlation $(r=0.328)$, hence function1 is important for the classification of the independent variables to the three risk groups. The chi-square values $(\chi 2=8.336)$ which is a statistics for measuring these tests of significance of the Eigen values; hence the test of the Eigen values is significant. The result shows there is significant relationship between the discriminant function 1 and the independent variables of c15 related groups. The discriminant function equation for predicting the classification of risk 3 with risk management techniques in software devotement project are given as:

$\mathrm{DF} 1=2.756 * \mathrm{c} 15-7.796$

R4: Risk of 'Insufficient/Inappropriate Staffing' Compared to Controls.

Table 7. Wilks' Lambda

\begin{tabular}{|l|r|r|r|r|}
\hline Test of Function(s) & Wilks' Lambda & Chi-square & Df & Sig. \\
\hline 1 through 2 & .542 & 43.509 & 10 & .000 \\
2 & .938 & 4.511 & 4 & .341 \\
\hline
\end{tabular}

Table 8. Classification Results for Risk 4 using Discriminant Function

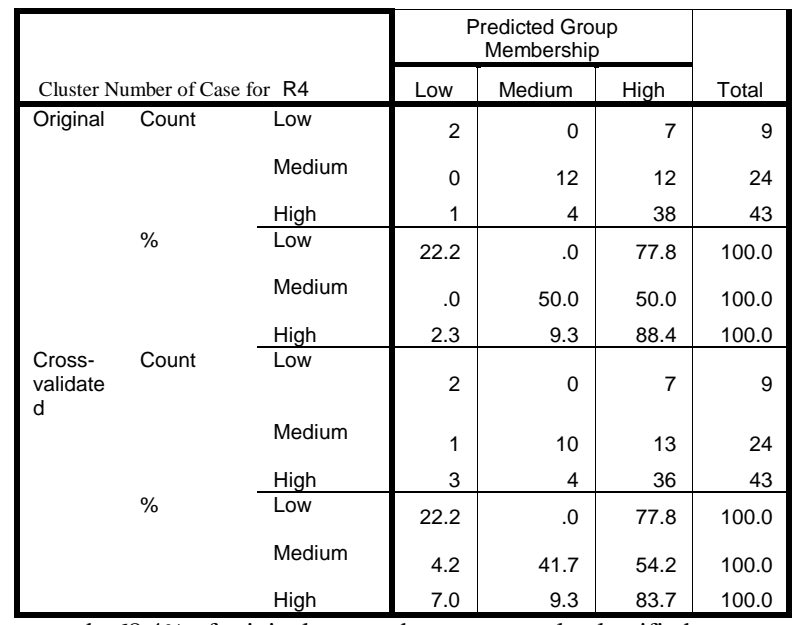

b $68.4 \%$ of original grouped cases correctly classified 
Table 7 and Table 8 show that discriminant function 1 explains $91.8 \%$ of the variance between the risk groups. Also it indicates the canonical correlation of the discriminant functions to the independent variables. Functions 1 has positive correlation $(\mathrm{r}=0.650)$ higher than the chi-square values $\left(\chi^{2}=43.509\right)$ which is a statistics for measuring these tests of significance of the Eigen values. The result shows there is significant relationship between the discriminant function 1 and the independent variables of c1, c3, c4, c12, c13 related groups. The discriminant function equation for predicting the classification of risk 4 with risk management techniques in software devotement project are given as:

$$
\mathrm{DF} 1=-1.610 * \mathrm{c} 1-1.112 * \mathrm{c} 3+1.658 * \mathrm{c} 4+1.153 * \mathrm{c} 12+1.198 * \mathrm{c} 13-3.376
$$

\section{R5: Risk of 'Lack of Senior Management Commitment and Technical Leadership' Compared to Controls.}

Table 9. Wilks' Lambda

\begin{tabular}{|r|r|r|r|r|}
\hline Test of Function(s) & Wilks' Lambda & Chi-square & df & Sig. \\
\hline 1 through 2 & .764 & 19.485 & 4 & .001 \\
2 & .933 & 4.999 & 1 & .025 \\
\hline
\end{tabular}

\section{Table 10. Classification Results for Risk 5 using Discriminant Function}

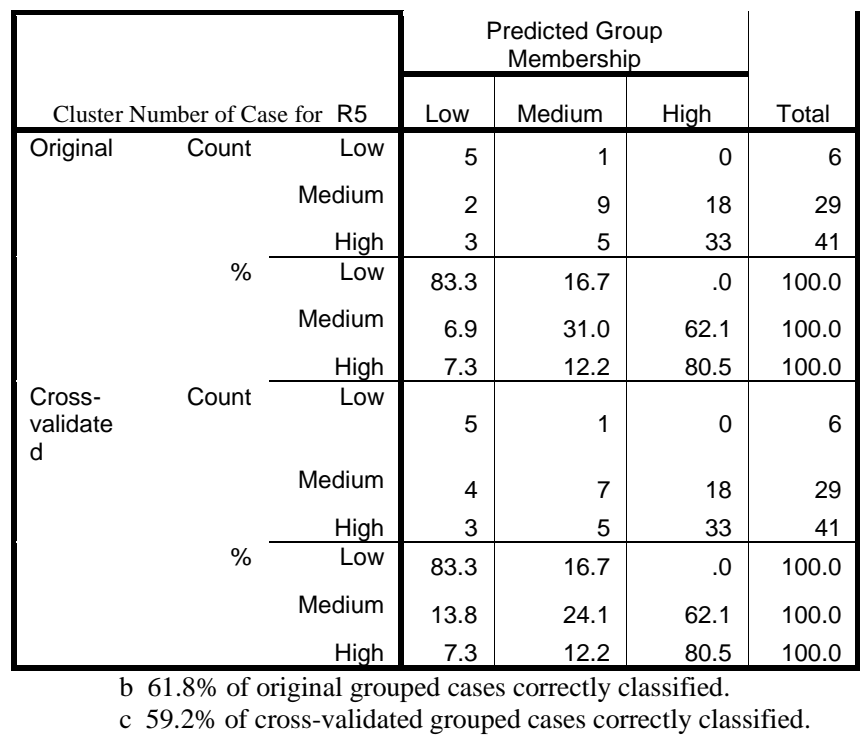

Table 9 and Table 10 show that discriminant function 1 explains $75.6 \%$ of the variance between the risk groups while discriminant function 2 only accounts for $24.4 \%$ of the variance. Functions 1 and 2 have positive correlation $(r=0.426$, 0.258 ), hence both function 1 and 2 are important for the classification of the independent variables to the risk groups. The chi-square values $(\chi 2=19.485,4.999)$ which is a statistics for measuring these tests of significance of the Eigen values, hence both test of the Eigen values are significant. The result shows there is significant relationship between the discriminant function 1 and 2 the independent variables of c6, $\mathrm{c} 7$ related groups. From the results in equation the two discriminant function equations for predicting the classification of risk 5 with risk management techniques in software devotement project are given as: 
DF1 $=2.542 * \mathrm{c} 6-1.263 * \mathrm{c} 7-3.484$
DF2 $=-.032 * \mathrm{c} 6+2.149 * \mathrm{c} 7-5.877$

R6: Risk of 'Poor /Inadequate Software Project Planning and Strategic Thinking' Compared to Controls.

Table 11. Wilks' Lambda

\begin{tabular}{|l|r|r|r|r|}
\hline Test of Function(s) & Wilks' Lambda & Chi-square & \multicolumn{1}{c|}{ df } & Sig. \\
\hline 1 & .914 & 6.593 & 2 & .037 \\
\hline
\end{tabular}

Table 12. Classification Results for Risk 6 using Discriminant Function

\begin{tabular}{|c|c|c|c|c|c|c|}
\hline \multirow{2}{*}{\multicolumn{3}{|c|}{ Cluster Number of Case for R6 }} & \multicolumn{3}{|c|}{ Predicted Group Membership } & \multirow[b]{2}{*}{ Total } \\
\hline & & & Low & Medium & High & \\
\hline \multirow[t]{6}{*}{ Original } & Count & Low & 0 & 1 & 2 & 3 \\
\hline & & Medium & 0 & 9 & 12 & 21 \\
\hline & & High & 0 & 7 & 45 & 52 \\
\hline & $\%$ & Low & .0 & 33.3 & 66.7 & 100.0 \\
\hline & & Medium & .0 & 42.9 & 57.1 & 100.0 \\
\hline & & High & .0 & 13.5 & 86.5 & 100.0 \\
\hline \multirow{6}{*}{$\begin{array}{l}\text { Cross- } \\
\text { validated }\end{array}$} & Count & Low & 0 & 1 & 2 & 3 \\
\hline & & Medium & 0 & 9 & 12 & 21 \\
\hline & & High & 0 & 7 & 45 & 52 \\
\hline & $\%$ & Low & .0 & 33.3 & 66.7 & 100.0 \\
\hline & & Medium & .0 & 42.9 & 57.1 & 100.0 \\
\hline & & High & .0 & 13.5 & 86.5 & 100.0 \\
\hline
\end{tabular}

Functions 1 has positive correlation $(r=0.294)$. The chi-square values $\left(\chi^{2}=\right.$ 6.596) which is a statistics for measuring these tests of significance of the Eigen values, hence both test of the Eigen values are significant. The result shows there is significant relationship between the discriminant function 1 and the independent variables of $\mathrm{c} 1$ related groups. From the results in equation the discriminant function equations for predicting the classification of risk 6 with risk management techniques in software devotement project are given as:

$\mathrm{DF} 1=2.092 * \mathrm{c} 1-5.754$

R7: Risk of 'Lack of An Effective Software Project Management Methodology' Compared to Controls.

Table 13. Wilks' Lambda

\begin{tabular}{|l|r|r|r|c|}
\hline Test of Function(s) & Wilks' Lambda & Chi-square & df & Sig. \\
\hline 1 through 2 & .684 & 27.332 & 6 & .000 \\
2 & .882 & 9.056 & 2 & .011 \\
\hline
\end{tabular}


Table 14. Classification Results for Risk 7 using Discriminant Function

\begin{tabular}{|c|c|c|c|c|c|c|}
\hline & & & \multicolumn{3}{|c|}{$\begin{array}{l}\text { Predicted Group } \\
\text { Membership }\end{array}$} & \multirow[b]{2}{*}{ Total } \\
\hline \multicolumn{3}{|c|}{ Cluster Number of Case for R7 } & Low & Medium & High & \\
\hline \multirow[t]{6}{*}{ Original } & Count & Low & 3 & 0 & 0 & 3 \\
\hline & & Medium & 3 & 11 & 14 & 28 \\
\hline & & High & 3 & 5 & 37 & 45 \\
\hline & $\%$ & Low & 100.0 & .0 & .0 & 100.0 \\
\hline & & Medium & 10.7 & 39.3 & 50.0 & 100.0 \\
\hline & & High & 6.7 & 11.1 & 82.2 & 100.0 \\
\hline \multirow{6}{*}{$\begin{array}{l}\text { Cross- } \\
\text { validated( } \\
\text { a) }\end{array}$} & Count & Low & 0 & 1 & 2 & 3 \\
\hline & & Medium & 3 & 11 & 14 & 28 \\
\hline & & High & 3 & 5 & 37 & 45 \\
\hline & $\%$ & Low & .0 & 33.3 & 66.7 & 100.0 \\
\hline & & Medium & 10.7 & 39.3 & 50.0 & 100.0 \\
\hline & & High & 6.7 & 11.1 & 82.2 & 100.0 \\
\hline
\end{tabular}

b $67.1 \%$ of original grouped cases correctly classified.

c $63.2 \%$ of cross-validated grouped cases correctly classified.

Table 13 and Table 14 show that discriminant function 1 explains $68.3 \%$ of the variance between the risk groups while discriminant function 2 only accounts for $31.7 \%$ of the variance. Functions 1 and 2 have positive correlation $(\mathrm{r}=0.473$, $0.344)$. Eigen value is significant for the discriminant function 1 and 2 . The chisquare values $(\chi 2=27.332,9.056)$ which is a statistics for measuring these tests of significance of the Eigen values, hence both test of the Eigen values are significant. The result shows there is significant relationship between the discriminant functions and the independent variables of $\mathrm{c} 9, \mathrm{c} 11$, and $\mathrm{c} 24$ related groups. The discriminant function for predicting the classification of risk 7 with risk management techniques in software devotement project are given as:

$$
\begin{aligned}
& \mathrm{DF} 1=2.039 * \mathrm{c} 9-2.439 * \mathrm{c} 11+1.253 * \mathrm{c} 24-2.440 \\
& \mathrm{DF} 2=0.072 * \mathrm{c} 9+.888 * \mathrm{c} 11+2.116 * \mathrm{c} 24-8.636
\end{aligned}
$$

R8: Risk of 'Change in Organizational Management During The Software Project' Compared to Controls.

Table 15. Wilks' Lambda

\begin{tabular}{|c|c|c|c|c|}
\hline Test of Function(s) & $\begin{array}{c}\text { Wilks' } \\
\text { Lambda }\end{array}$ & Chi-square & df & Sig. \\
\hline 1 & .877 & 9.615 & 2 & .008 \\
\hline
\end{tabular}




\section{Table 16. Classification Results for Risk 8 using Discriminant Function}

\begin{tabular}{|c|c|c|c|c|c|c|}
\hline \multirow{2}{*}{\multicolumn{3}{|c|}{ Cluster Number of Case for R8 }} & \multicolumn{3}{|c|}{ Predicted Group Membership } & \multirow[b]{2}{*}{ Total } \\
\hline & & & Low & Medium & High & \\
\hline \multirow[t]{2}{*}{ Original } & Count & $\begin{array}{c}\text { Low } \\
\text { Medium } \\
\text { High }\end{array}$ & $\begin{array}{l}0 \\
0 \\
0\end{array}$ & $\begin{array}{l}3 \\
7 \\
3\end{array}$ & $\begin{array}{c}3 \\
18 \\
42\end{array}$ & $\begin{array}{c}6 \\
25 \\
45\end{array}$ \\
\hline & $\%$ & $\begin{array}{l}\text { Low } \\
\text { Medium } \\
\text { High }\end{array}$ & $\begin{array}{l}.0 \\
.0 \\
.0\end{array}$ & $\begin{array}{c}50.0 \\
28.0 \\
6.7 \\
\end{array}$ & $\begin{array}{l}50.0 \\
72.0 \\
93.3 \\
\end{array}$ & $\begin{array}{l}100.0 \\
100.0 \\
100.0 \\
\end{array}$ \\
\hline \multirow[t]{3}{*}{$\begin{array}{c}\text { Cross- } \\
\text { validated }\end{array}$} & Count & Low & 0 & 3 & 3 & 6 \\
\hline & & $\begin{array}{l}\text { Medium } \\
\text { High }\end{array}$ & $\begin{array}{l}2 \\
0\end{array}$ & $\begin{array}{l}5 \\
3\end{array}$ & $\begin{array}{l}18 \\
42\end{array}$ & $\begin{array}{l}25 \\
45\end{array}$ \\
\hline & $\%$ & $\begin{array}{l}\text { Low } \\
\text { Medium } \\
\text { High }\end{array}$ & $\begin{array}{c}.0 \\
8.0 \\
.0\end{array}$ & $\begin{array}{c}50.0 \\
20.0 \\
6.7\end{array}$ & $\begin{array}{l}50.0 \\
72.0 \\
93.3\end{array}$ & $\begin{array}{l}100.0 \\
100.0 \\
100.0\end{array}$ \\
\hline
\end{tabular}

b $64.5 \%$ of original grouped cases correctly classified.

c $61.8 \%$ of cross-validated grouped cases correctly classified.

Functions 1 has positive correlation $(r=0.350)$, hence function 1 is important for the classification of the independent variables to the risk groups. The chi-square values $\left(\chi^{2}=9.615\right)$ which is a statistics for measuring these tests of significance of the Eigen values; hence both test of the Eigen value is significant. There is significant relationship between the discriminant function 1 and the independent variables of c17 related groups. The discriminant function equations for predicting the classification of risk 8 with risk management techniques in software devotement project are given as:

$\mathrm{DF} 1=2.279 * \mathrm{C} 7-6.386$

R9: Risk of 'Ineffective Communication Software Project System' Compared to Controls.

Table 17. Wilks' Lambda

\begin{tabular}{|l|l|l|l|l|}
\hline Test of Function(s) & Wilks' Lambda & Chi-square & Df & Sig. \\
\hline 1 through 2 & .654 & 30.581 & 6 & .000 \\
2 & .995 & .328 & 2 & .849 \\
\hline
\end{tabular}

Table 18. Classification Results for Risk 9 using Discriminant Function

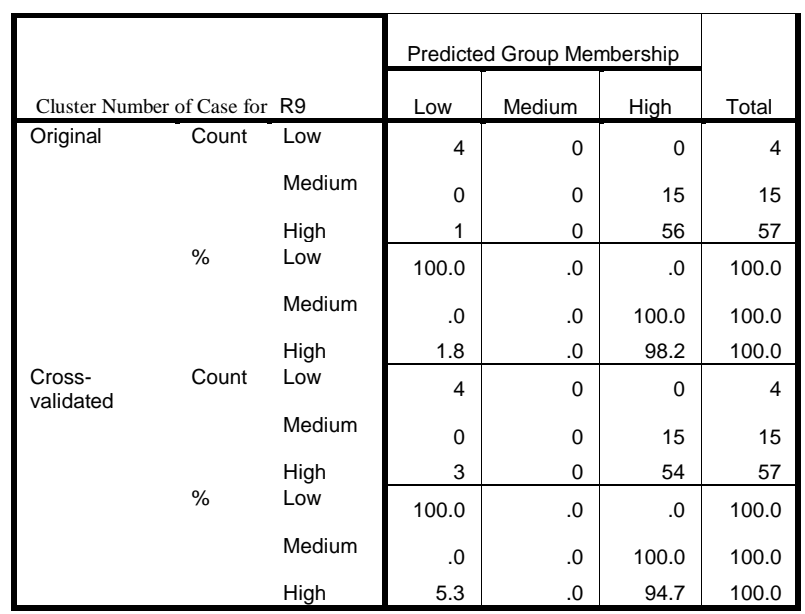

b $78.9 \%$ of original grouped cases correctly classified.

c $76.3 \%$ of cross-validated grouped cases correctly classified. 
Table 17 and Table 18 show that discriminant function 1 explains $99.1 \%$ of the variance between the risk groups. Also it indicates the canonical correlation of the discriminant functions to the independent variables. Functions 1 have positive correlation $(\mathrm{r}=0.586)$, hence both function1 is important for the classification of the independent variables to the risk groups. The chi-square values $\left(\chi^{2}=30.581\right)$ which is a statistics for measuring these tests of significance; hence both test of the Eigen values is significant. The result shows there is significant relationship between the discriminant function 1 and the independent variables of $\mathrm{c} 1, \mathrm{C} 6$, and C7 related groups. From the results in equation the two discriminant function equations for predicting the classification of risk 9 with risk management techniques in software devotement project are given as:

$$
\mathrm{DF} 1=1.620 * \mathrm{C} 1+1.620 * \mathrm{C} 6-2.067 * \mathrm{C} 7-3.172
$$

\section{R10: Risk of 'Absence of a Historical Data (Templates)' Compared to Controls.}

Table 19. Wilks' Lambda

\begin{tabular}{|l|r|r|r|c|}
\hline Test of Function(s) & Wilks' Lambda & Chi-square & \multicolumn{1}{c|}{ Df } & \multicolumn{1}{c|}{ Sig. } \\
\hline 1 & .818 & 14.706 & 2 & .001 \\
\hline
\end{tabular}

Table 20. Classification Results for Risk 10 using Discriminant Function

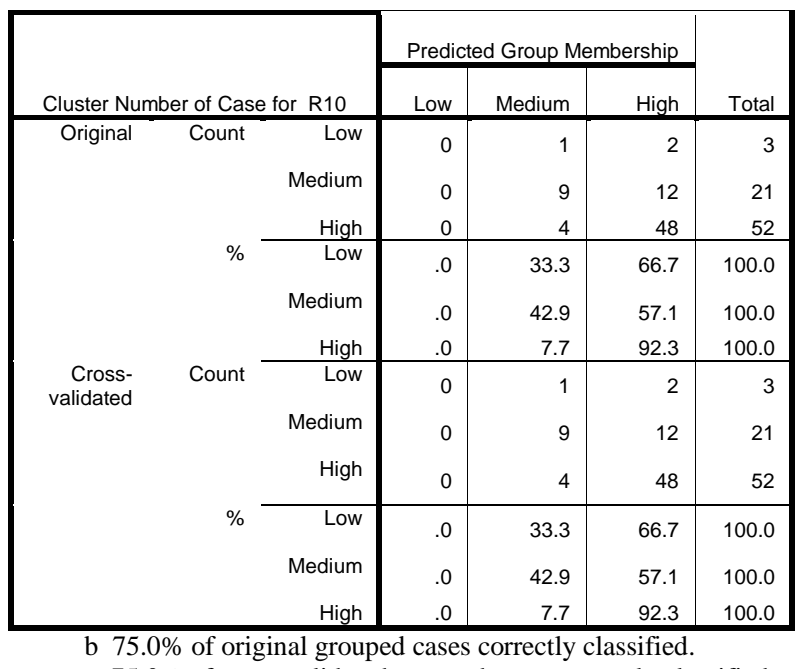

c $75.0 \%$ of cross-validated grouped cases correctly classified.

Functions 1 has positive correlation $(r=0.427)$, hence Function 1 is used for the classifying of the controls to the risk groups. The chi-square values $(\chi 2=14.706)$ which is measuring the significance of the Eigen values; hence both test of the Eigen values is significant. The result shows there is significant relationship between the discriminant function 1 and the independent variables of c5 related groups. From the results in equation the two discriminant function equations for predicting the classification of risk 10 with risk management techniques in software devotement project are given as:

$\mathrm{DF} 1=2.317 * \mathrm{c} 5-6.463$

\subsection{Identifying Software Planning Risks by using Control Techniques}

Table 21 illustrate risks were mitigating by using Control Techniques 
Table 21. Software Risk Planning Development Factors were Mitigated by using Control Techniques

\begin{tabular}{|c|c|c|}
\hline module & Software Risks & Risk Control Techniques \\
\hline 1 & Low key user involvement. & $\begin{array}{l}\text { C1: Using of requirements scrubbing, C21: } \\
\text { Including formal and periodic risk } \\
\text { assessment, C28: Maintain proper } \\
\text { documentation of each individual's work. }\end{array}$ \\
\hline 2 & Unrealistic schedules and budgets. & $\begin{array}{l}\text { C5: Developing and adhering a software } \\
\text { project plan. }\end{array}$ \\
\hline 3 & $\begin{array}{l}\text { Misunderstood /Unrealistic scope and } \\
\text { objectives (goals). }\end{array}$ & C15: Reusable user documents early. \\
\hline 4 & Insufficient/inappropriate staffing. & $\begin{array}{l}\text { C1: Using of requirements scrubbing, C3: } \\
\text { Assessing cost and scheduling the impact of } \\
\text { each change to requirements and } \\
\text { specifications, C4: Develop prototyping and } \\
\text { have the requirements reviewed by the } \\
\text { client, C12: Reusable source code and } \\
\text { interface methods, C13: Reusable test plans } \\
\text { and test cases. }\end{array}$ \\
\hline 5 & $\begin{array}{l}\text { Lack of senior management commitment } \\
\text { and technical leadership. }\end{array}$ & $\begin{array}{l}\text { C6: Implementing and following a } \\
\text { communication plan, C7: Developing } \\
\text { contingency plans to cope with staffing } \\
\text { problems. }\end{array}$ \\
\hline 6 & $\begin{array}{l}\text { Poor/inadequate software project } \\
\text { planning and strategic thinking. }\end{array}$ & $\mathrm{C} 1$ : Using of requirements scrubbing. \\
\hline 7 & $\begin{array}{l}\text { Lack of an effective software project } \\
\text { management methodology. }\end{array}$ & $\begin{array}{l}\text { C9: Have team-building sessions, C11: } \\
\text { Dividing the software project into } \\
\text { controllable portions, C24: Ensuring that } \\
\text { quality-factor deliverables and task analysis. }\end{array}$ \\
\hline 8 & $\begin{array}{l}\text { Change in organizational management } \\
\text { during the software project. }\end{array}$ & $\begin{array}{l}\text { C17: Implement/ utilize benchmarking and } \\
\text { tools of technical analysis. }\end{array}$ \\
\hline 9 & $\begin{array}{l}\text { Ineffective communication software } \\
\text { project system. }\end{array}$ & $\begin{array}{l}\text { C1: Using of requirements scrubbing, C6: } \\
\text { Implementing and following a } \\
\text { communication plan, C7: Developing } \\
\text { contingency plans to cope with staffing } \\
\text { problems. }\end{array}$ \\
\hline 10 & Absence of a historical data (templates). & $\begin{array}{l}\text { C5: Developing and adhering a software } \\
\text { project plan. }\end{array}$ \\
\hline
\end{tabular}

\section{Conclusions}

The paper is the classifying risks of software planning development. These statistics techniques were used the discriminant analysis techniques, to compare the controls to each of the risk factors to determine if they are effective in mitigating the occurrence of each risk factor. However, we classified software planning risks to high, medium, low by Predicted Group Membership. However, we are referred the controls in risk management approach were mitigated on software planning risk software development factors in Table 21. In future work, we can use more techniques useful to classify and predict software project risk models such as artificial intelligence techniques. 


\section{References}

[1] A. Elzamly and B. Hussin, "Estimating Stepwise and Fuzzy Regression Analysis for Modelling Software Design Project Risks," Asian J. Math. Comput. Res., vol. 3, no. 4, pp. 234-241, (2015).

[2] A. Elzamly and B. Hussin, "An Enhancement of Framework Software Risk Management Methodology for Successful Software Development”, Theor. Appl. Inf. Technol., vol. 62, no. 2, (2014), pp. 410-423.

[3] J. Sodhi and P. Sodhi, "IT Project Management Handbook", Management Concepts, (2001).

[4] K. Khanfar, A. Elzamly, W. Al-Ahmad, E. El-Qawasmeh, K. Alsamara and S. Abuleil, "Managing Software Project Risks with the Chi-Square Technique", Int. Manag. Rev., vol. 4, no. 2, (2008), pp. 1829.

[5] A. Elzamly and B. Hussin, "Managing Software Project Risks with Proposed Regression Model Techniques and Effect Size Technique", Int. Rev. Comput. Softw., vol. 6, no. 2, (2011), pp. 250-263.

[6] A. Elzamly and B. Hussin, "Estimating Quality-Affecting Risks in Software Projects", Int. Manag. Rev. Am. Sch. Press, vol. 7, no. 2, (2011), pp. 66-83.

[7] A. Elzamly and B. Hussin, "Managing Software Project Risks ( Implementation Phase ) with Proposed Stepwise Regression Analysis Techniques", Int. J. Inf. Technol., vol. 1, no. 4, (2013), pp. 300-312.

[8] A. Elzamly and B. Hussin, "Managing Software Project Risks (Design Phase) with Proposed Fuzzy Regression Analysis Techniques with Fuzzy Concepts," Int. Rev. Comput. Softw., vol. 8, no. 11, (2013), pp. 2601-2613.

[9] A. Elzamly and B. Hussin, "A Comparison of Fuzzy and Stepwise Multiple Regression Analysis Techniques for Managing Software Project Risks : Implementation Phase”, Int. Manag. Rev., vol. 10, no. 1, (2014), pp. 43-54.

[10] A. Elzamly, B. Hussin and N. Salleh, "Methodologies and techniques in software risk management approach for mitigating risks: A review", Asian J. Math. Comput. Res., vol. 2, no. 4, (2015), pp. 184198.

[11] A. Elzamly and B. Hussin, "Modelling and Evaluating Software Project Risks with Quantitative Analysis Techniques in Planning", Comput. Inf. Technol., vol. 23, no. 2, (2015), pp. 113-120.

[12] A. Elzamly and B. Hussin, "Managing Software Project Risks (Planning Phase) with Proposed Fuzzy Regression Analysis Techniques with Fuzzy Concepts", Int. J. Inf. Comput. Sci., vol. 3, no. 2, (2014), pp. 31-40.

[13] A. Elzamly and B. Hussin, "Managing Software Project Risks (Analysis Phase) with Proposed Fuzzy Regression Analysis Modelling Techniques with Fuzzy Concepts", J. Comput. Inf. Technol., vol. 22, no. 2, (2014), pp. 131-144.

[14] A. Elzamly and B. Hussin, "Evaluation of Quantitative and Mining Techniques for Reducing Software Maintenance Risks", Appl. Math. Sci., vol. 8, no. 111, (2014), pp. 5533-5542.

[15] A. Elzamly and B. Hussin, "Modelling and mitigating Software Implementation Project Risks with Proposed Mining Technique", Inf. Eng., vol. 3, (2014), pp. 39-48.

[16] A. Elzamly and B. Hussin, "A Comparison of Stepwise And Fuzzy Multiple Regression Analysis Techniques for Managing Software Project Risks: Analysis Phase”, Comput. Sci., vol. 10, no. 10, (2014), pp. 1725-1742.

[17] A. Elzamly and B. Hussin, "Mitigating Software Maintenance Project Risks with Stepwise Regression Analysis Techniques", Mod. Math. Front., vol. 3, no. 2, (2014), pp. 34-44.

[18] A. Elzamly and B. Hussin, "Classification and identification of risk management techniques for mitigating risks with factor analysis technique in software risk management”, Rev. Comput. Eng. Res., vol. 2, no. 1, (2015), pp. 22-38.

[19] A. Elzamly and B. Hussin, "Identifying and Managing Software Project Risks with Proposed Fuzzy Regression Analysis Techniques: Maintenance Phase", 2014 Conference on Management and Engineering (CME2014), (2014), pp. 1868-1881. 
International Journal of Advanced Science and Technology Vol.81 (2015) 